To appear in Mathematical Methods and Models in the Applied Sciences 12 (2002)

\title{
NONCONFORMING MIXED ELEMENTS FOR ELASTICITY
}

\author{
DOUGLAS N. ARNOLD AND RAGNAR WINTHER
}

Dedicated to Jim Douglas, Jr., on the occasion of this 75th birthday.

\begin{abstract}
We construct first order, stable, nonconforming mixed finite elements for plane elasticity and analyze their convergence. The mixed method is based on the HellingerReissner variational formulation in which the stress and displacement fields are the primary unknowns. The stress elements use polynomial shape functions but do not involve vertex degrees of freedom.
\end{abstract}

\section{INTRODUCTION}

Mixed finite element methods for linear elasticity are based on approximations of a stressdisplacement system derived from the Hellinger-Reissner variational principle. If $\sigma$ and $u$ denote the stress and displacement fields engendered by a body force $f$ acting on a linearly elastic body which occupies a planar region $\Omega$, and which is clamped at the boundary $\partial \Omega$, then the pair $(\sigma, u)$ can be characterized as the unique critical point of the functional

$$
\mathcal{J}(\tau, v)=\int_{\Omega}\left(\frac{1}{2} A \tau: \tau+\operatorname{div} \tau \cdot v-f \cdot v\right) d x .
$$

Here $\sigma$ takes values in the space $\mathbb{S}=\mathbb{R}_{\text {sym }}^{2 \times 2}$ of symmetric tensors and $u$ in $\mathbb{R}^{2}$, while the given compliance tensor $A=A(x): \mathbb{S} \rightarrow \mathbb{S}$ is bounded and symmetric positive definite uniformly for $x \in \Omega$.

A mixed finite element method requires the construction of a pair of finite element spaces, $\Sigma_{h}$ and $V_{h}$, for the approximation of the stress and the displacement, which are properly related in the sense that they satisfy the stability conditions for mixed methods [6, 7]. Although there are number of well-known stable mixed finite element pairs known for the analogous problem involving vector fields and scalar fields, the combination of the symmetry and continuity conditions of the stress field represents a substantial additional difficulty. In fact, until recently the only stable pairs known used composite elements, in which $V_{h}$ consists of piecewise polynomials with respect to one triangulation of the domain, while $\Sigma_{h}$ consists of piecewise polynomials with respect to a different, more refined, triangulation $[3,9,10,16]$.

In a recent paper [5] we constructed a new family of stable elements for the HellingerReissner formulation. These spaces consist of piecewise polynomials with respect to a single arbitrary triangular subdivision of $\Omega$. The simplest and lowest order spaces in this family consist of discontinuous piecewise linear vector fields for velocity, while the space $\Sigma_{h}$ consists locally of the span of piecewise quadratic matrix fields and the cubic matrix fields which are

1991 Mathematics Subject Classification. Primary: 65N30, Secondary: 74S05.

Key words and phrases. mixed method, nonconforming finite element, elasticity.

The first author was supported by NSF grant DMS-9870399 and the second author by the Research Council of Norway under grant 135420/431. 
divergence-free. Hence, it takes 24 stess and 6 displacement degrees of freedom to determine an element of $\Sigma_{h} \times V_{h}$ on a given triangle. We also constructed a simpler first order element pair with 21 stress and 3 displacement degrees of freedom per triangle. All these elements involve vertex degrees of freedom for the stress field. This implies continuity of the stress at the vertices, which is not required by the mixed variational principle, and is undesireable for certain implementational approaches. However, as shown in [5], vertex degrees of freedom are unavoidable for conforming finite elements with continuous shape functions.

Motivated by the unavoidable complexity of conforming mixed elements, and, in particular, their requirement of vertex degrees of freedom, in this paper we construct and analyze some simpler nonconforming elements. First, we present an element with 15 local degrees of freedom for the stress and 6 for the displaceent. This element is closely related to the composite finite element method proposed in [16] and [10]. In fact, the degrees of freedom are identical. For both methods the dimension of the discrete system is $9|T|+4|e|$, where $|T|$ and $|e|$ denotes the number of triangles and edges of the triangulation, respectively. However, for our element there is no need for a refined triangulation to represent the discrete stress shape functions. For the second element we propose, the local degrees of freedom for $\Sigma_{h}$ and $V_{h}$ are further reduced to 12 and 3, respectively, while the global dimension is reduced to $3|T|+4|e|$. Hence, the size of the discrete system is reduced by approximately $40 \%$. For this reduced element the velocity space is simply the space of piecewise rigid motions.

In the original continuous problem the critical point $(\sigma, u)$ of the Hellinger-Reissner fuctional $\mathcal{J}$ is sought among all $(\tau, v) \in H(\operatorname{div}, \Omega, \mathbb{S}) \times L^{2}\left(\Omega, \mathbb{R}^{2}\right)$. Here $H(\operatorname{div}, \Omega, \mathbb{S})$ denotes the space of square-integrable symmetric matrix fields with square-integrable divergence, while $L^{2}\left(\Omega, \mathbb{R}^{2}\right)$ is the space of square-integrable vector fields. Equivalently, $(\sigma, u) \in$ $H(\operatorname{div}, \Omega, \mathbb{S}) \times L^{2}\left(\Omega, \mathbb{R}^{2}\right)$ is the unique solution to the following weak formulation of the elasticity system:

$$
\int_{\Omega}(A \sigma: \tau+\operatorname{div} \tau \cdot u+\operatorname{div} \sigma \cdot v) d x=\int_{\Omega} f \cdot v d x, \quad(\tau, v) \in H(\operatorname{div}, \Omega, \mathbb{S}) \times L^{2}\left(\Omega, \mathbb{R}^{2}\right) .
$$

Once $\Sigma_{h} \subset H(\operatorname{div}, \Omega, \mathbb{S})$ and $V_{h} \subset L^{2}\left(\Omega, \mathbb{R}^{2}\right)$ are chosen, the corresponding conforming mixed finite element method determines an approximate stress field $\sigma_{h}$ and an approximate displacement field $u_{h}$ as the critical point of $\mathcal{J}$ over $\Sigma_{h} \times V_{h}$. Equivalently, the pair $\left(\sigma_{h}, u_{h}\right) \in$ $\Sigma_{h} \times V_{h}$ is determined by the weak formulation (1.2), with the test space restricted to $\Sigma_{h} \times V_{h}$.

The methods discussed in this paper are nonconforming in that the trial space $\Sigma_{h}$ is not contained in $H(\operatorname{div}, \Omega, \mathbb{S})$ because the required continuity of the normal component across element edges is not imposed. The functional $\mathcal{J}$ is extended to $\Sigma_{h} \times V_{h}$ by replacing the divergence operator by $\operatorname{div}_{h}$, the divergence applied element by element, and then the approximate solution $\left(\sigma_{h}, u_{h}\right)$ is determined as the critical point of the extended functional over $\Sigma_{h} \times V_{h}$. As we shall see, sufficient interelement continuity is enforced to ensure convergence of the method.

As in the conforming case, the subspaces $\Sigma_{h}$ and $V_{h}$ have to be properly related. To ensure that a unique critical point exist and that it provides a good approximation of the true solution, they must satisfy the stability conditions from the theory of mixed methods $[6,7]$. Hence, there should exist positive constants $c_{1}, c_{2}$, independent of the mesh parameter $h$, such that

(A1) If $\tau \in \Sigma_{h}$ satisfies $\int_{\Omega} \operatorname{div}_{h} \tau \cdot v d x=0$ for all $v \in V_{h}$ then $\|\tau\|_{H\left(\operatorname{div}_{h}\right)} \leq c_{1}\|\tau\|_{L^{2}}$. 
(A2) For all $v \in V_{h}$ there exists nonzero $\tau \in \Sigma_{h}$ with $\int_{\Omega} \operatorname{div}_{h} \tau \cdot v d x \geq c_{2}\|\tau\|_{H\left(\operatorname{div}_{h}\right)}\|v\|_{L^{2}}$. Here $\|\tau\|_{H\left(\operatorname{div}_{h}\right)}=\left(\|\tau\|_{L^{2}}^{2}+\left\|\operatorname{div}_{h} \tau\right\|_{L^{2}}^{2}\right)^{1 / 2}$.

The lack of simple stables choices of mixed elements for the Hellinger-Reissner functional has lead to the construction of a collection of methods based on modified variational principles in which the symmetry of the stress tensor is enforced only weakly or abandoned altogether. Methods of this type are discussed, for example, in $[1,2,4,12,13,14,15]$. See also the discussion in [5].

\section{Notation And PRELIMINARIES}

We denote by $H^{k}(T, X)$ the Sobolev space consisting of functions with domain $T \subset \mathbb{R}^{2}$, taking values in the finite-dimensional vector space $X$, and with all derivatives of order at most $k$ square-integrable. For our purposes, the range space $X$ will be either $\mathbb{S}, \mathbb{R}^{2}$, or $\mathbb{R}$, and in the latter case we write simply $H^{k}(T)$. We will generally write $\|\cdot\|_{k}$ instead of $\|\cdot\|_{H^{k}(T, X)}$. We similarly denote by $\mathcal{P}_{k}(T, X)$ the space of polynomials on $T$ with degree at most $k$.

The symmetric part of the gradient of a vector field $v$, denoted $\epsilon v$, is given by $\epsilon v=$ $\left[\operatorname{grad} v+(\operatorname{grad} v)^{T}\right] / 2$. If $\tau$ is a symmetric matrix field then its divergence, $\operatorname{div} \tau$, is the vector field obtained by applying the ordinary divergence operator to each row. Hence, if $\tau$ is piecewise smooth matrix field then $\tau \in H(\operatorname{div}, \Omega, \mathbb{S})$ if and only if $\tau n$ is continuous across the interface, where $n$ is a nonzero vector normal to the interface. The Airy stress function associated to a scalar field $q$ is given by

$$
J q=\left(\begin{array}{cc}
\partial^{2} q / \partial y^{2} & -\partial^{2} q / \partial x \partial y \\
-\partial^{2} q / \partial x \partial y & \partial^{2} q / \partial x^{2}
\end{array}\right)
$$

Note that, on $\partial \Omega$

$$
(J q) n=\frac{\partial}{\partial s} \operatorname{curl} q, \quad n^{T}(J q) n=\frac{\partial^{2} q}{\partial s^{2}}, \quad t^{T}(J q) n=-\frac{\partial^{2} q}{\partial s \partial n},
$$

where $\operatorname{curl} q=(-\partial q / \partial y, \partial q / \partial x)$ and $n$ and $t$ are the unit normal and tangent vectors.

Throughout the paper we assume that the elastic domain $\Omega$ is a simply connected polygon. Any smooth vector field on $\Omega$ may be realized as the divergence of a smooth symmetric matrix field. E.g., we may extend the vector field smoothly to a larger smoothly bounded domain, and then solve the equations of elasticity there with the extended vector field as body forces. The same argument shows that any vector field in $L^{2}\left(\Omega, \mathbb{R}^{2}\right)$ may be realized as the divergence of a matrix field in $H^{1}(\Omega, \mathbb{S})$, a fact we will use below. Further, a smooth symmetric matrix field $\tau$ on a simply connected domain is divergence-free if and only if $\tau=J q$ for some smooth function $q$. The potential $q$ is determined by $\tau$ up to addition of a linear polynomial. These considerations are summarized by the statement that the following sequence is exact (i.e., that the range of each map is the kernel of the following one):

$$
0 \longrightarrow \mathcal{P}_{1}(\Omega) \stackrel{\subset}{\longrightarrow} C^{\infty}(\Omega) \stackrel{J}{\longrightarrow} C^{\infty}(\Omega, \mathbb{S}) \stackrel{\operatorname{div}}{\longrightarrow} C^{\infty}\left(\Omega, \mathbb{R}^{2}\right) \longrightarrow 0 .
$$

We remark that this exact sequence is related, although rather indirectly, to the de Rham sequence for the domain the $\Omega$; see [8]. We have stated it in terms of infinitely differentiable functions, but analogous results hold with less smoothness. E.g., the sequence

$$
0 \longrightarrow \mathcal{P}_{1}(\Omega) \stackrel{\subset}{\longrightarrow} H^{2}(\Omega) \stackrel{J}{\longrightarrow} H(\operatorname{div}, \Omega, \mathbb{S}) \stackrel{\operatorname{div}}{\longrightarrow} L^{2}\left(\Omega, \mathbb{R}^{2}\right) \longrightarrow 0
$$


is also exact. There is a polynomial analogue of the sequence as well: for any integer $k \geq 0$ the sequence

$$
0 \longrightarrow \mathcal{P}_{1}(\Omega) \stackrel{\subset}{\longrightarrow} \mathcal{P}_{k+3}(\Omega) \stackrel{J}{\longrightarrow} \mathcal{P}_{k+1}(\Omega, \mathbb{S}) \stackrel{\operatorname{div}}{\longrightarrow} \mathcal{P}_{k}\left(\Omega, \mathbb{R}^{2}\right) \longrightarrow 0
$$

is exact. (To verify the surjectivity of the final divergence, it suffices to count dimensions and use the exactness of the sequence at the other points.) As was explained in [5], a key ingredient in the development and analysis of mixed methods for elasticity is a discrete analogue of the exact sequence (2.1) which is appropriately related to it. As we shall see below, both the methods studied here admit such an exact sequence.

\section{The Finite ELEMENT SPACES}

In section we present our first nonconforming finite element method. First we describe the finite elements on a single triangle $T \subset \Omega$. Define

$$
\begin{gathered}
\Sigma_{T}=\left\{\tau \in \mathcal{P}_{2}(T, \mathbb{S}) \mid n \cdot \tau n \in \mathcal{P}_{1}(e), \quad \text { for each edge } e \text { of } T\right\}, \\
V_{T}=\mathcal{P}_{1}\left(T, \mathbb{R}^{2}\right) .
\end{gathered}
$$

Here, $n$ is a unit normal vector to $e$. The space $V_{T}$ has dimension 6 and a complete set of degrees of freedom are given by the value of the two components at the three nodes interior to $T$. The space $\Sigma_{T}$ clearly has dimension at least 15 , since the $\operatorname{dim} \mathcal{P}_{2}(T, \mathbb{S})=18$ and the condition that $n \cdot \tau n \in \mathcal{P}_{1}(e)$ on each edge represents three linear constraints. We now exhibit 15 degrees of freedom $\Sigma_{T} \rightarrow \mathbb{R}$ and show that they vanish simultaneously only when $\tau=0$. This implies that the dimension of $\Sigma_{T}$ is precisely 15, and that the degrees of freedom are unisolvent. The degrees of freedom are

- the values of the moments of degree 0 and 1 of the two normal components of $\tau$ on each edge $e$ of $T$ (12 degrees of freedom)

- the value of the three components of the moment of degree 0 of $\tau$ on $T$ (3 degrees of freedom)

Otherwise stated, we determine $\tau \in \Sigma_{T}$ by giving the values of $\int_{e}(\tau n) d s$ and $\int_{e}(\tau n) s d s$ for all edges, and the value of $\int_{T} \tau d x$. (Here $s$ is a parameter giving the distance to one of the end points of $e$.) Note that the degrees of freedom associated to an edge do not determine $\tau n$ on that edge. Therefore, this approach will not lead to a conforming approximation of $H(\operatorname{div}, \Omega, \mathbb{S})$. However, the component $n \cdot \tau n$ is determined by the degrees of freedom associated the edge, and also the two lowest order moments of $t \cdot \tau n$, where $t$ is a unit vector tangential to $e$. As we shall see below, this weak continuity of the functions in the finite element space is sufficient to define a convergent nonconforming finite element method.

The element diagrams in Figure 1 are mnemonic of the degrees of freedom.

Lemma 3.1. If the 15 degrees of freedom just given all vanish for some $\tau \in \Sigma_{T}$, then $\tau=0$.

Proof. For any $v \in \mathcal{P}_{1}(T, \mathbb{S})$ we have

$$
\int_{T}(\operatorname{div} \tau) \cdot v d x=-\int_{T} \tau: \epsilon v d x+\int_{\partial T} \tau n \cdot v d s .
$$

However, from the degrees of freedom we conclude that both integrals on the right hand side are zero. Thus $\tau$ is divergence-free and hence $\tau=J q$ for some $q \in \mathcal{P}_{4}(T)$; cf. (2.2). Adjusting by a linear function we may take $q$ to vanish at the vertices. Now $\partial^{2} q / \partial s^{2}=\tau n \cdot n=0$ on 

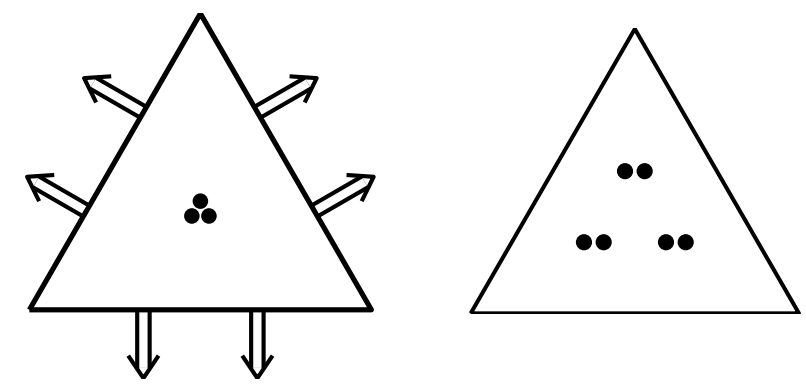

FigurE 1. Element diagrams for the stress and displacement elements.

each edge, whence $q$ is identically zero on $\partial T$. This implies that $q=p b_{T}$, where $p \in \mathcal{P}_{1}(T)$ and $b_{T}$ is the cubic bubble function on $T$ (the unique cubic polynomial achieving a maximum value of unity on $T$ and vanishing on $\partial T$ ). Hence, on each edge $e$,

$$
\frac{\partial q}{\partial n}=p \frac{\partial b_{T}}{\partial n}=p b_{e}
$$

where $b_{e}$ is a quadratic polynomial on $e$ vanishing at its endpoints. Since $-\tau n \cdot t=$ $\partial^{2} q / \partial s \partial n=\partial\left(p b_{e}\right) / \partial s$ is orthogonal to $\mathcal{P}_{1}(e)$, we must have that $p b_{e}$ is orthogonal to $\mathcal{P}_{0}(e)$, and therefore $p$ has a root in the interior of each edge $e$. However, since $p$ is linear this implies that $p \equiv 0$, and hence $\tau \equiv 0$.

Having given a unisolvent set of degrees of freedom for $V_{T}$ and $\Sigma_{T}$, our finite element space is assembled in the usual way. Let $\left\{\mathcal{T}_{h}\right\}$ be a family of shape regular triangulations of $\Omega$, , i.e., each $\mathcal{T}_{h}$ is a set of closed triangles with union $\bar{\Omega}$ and such that any two distinct non-disjoint elements of $\mathcal{T}_{h}$ meet in a common edge or vertex. The parameter $h$ is the maximal diameter of the triangles in $\mathcal{T}_{h}$. To each triangulation $\mathcal{T}_{h}$ the associated finite element element space $V_{h}$ is then the space of all piecewise linear vector fields with respect to this triangulation, not subject to any interelement continuity conditions. The space $\Sigma_{h}$ is the space of all matrix fields which belong piecewise to $\Sigma_{T}$, subject to the continuity conditions that the two lowest order moments of the normal components are continuous across mesh edges. Of course this condition is not sufficient to imply that $\Sigma_{h}$ is a subset of $H(\operatorname{div}, \Omega, \mathbb{S})$, since $t \cdot \tau n$ is not continuous across edges. Therefore, this choice of spaces leads to a nonconforming finite element discretization of the elasticity system (1.2).

The global degrees of freedom for the assembled finite element space $\Sigma_{h}$ are the moments of degrees 0 and 1 of the normal components on all the mesh edges, and the values of the moments of degree 0 for all components on all the mesh triangles. These functionals defines a corresponding interpolation operator $\Pi_{h}: H^{1}(\Omega, \mathbb{S}) \rightarrow \Sigma_{h}$ by

$$
\begin{gathered}
\int_{e}\left(\tau-\Pi_{h} \tau\right) n \cdot v d s=0 \quad \text { for all edges } e \text { and all } v \in \mathcal{P}_{1}\left(e, \mathbb{R}^{2}\right), \\
\int_{T}\left(\tau-\Pi_{h} \tau\right) d x=0 \text { for all triangles } T
\end{gathered}
$$

We have for any $\tau \in H^{1}(\Omega, \mathbb{S}), T \in \mathcal{T}_{h}$, and $v \in V_{T}$, that

$$
\int_{T} \operatorname{div}\left(\tau-\Pi_{h} \tau\right) \cdot v d x=-\int_{T}\left(\tau-\Pi_{h} \tau\right): \epsilon v d x+\int_{\partial T}\left(\tau-\Pi_{h} \tau\right) n \cdot v d s .
$$




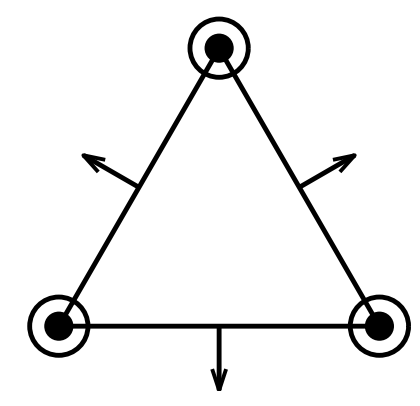

Figure 2. A nonconforming approximation of $H^{2}(\Omega)$.

The right hand side vanishes in view of (3.3) and (3.4). This verifies the commutativity property

$$
\operatorname{div}_{h} \Pi_{h} \tau=P_{h} \operatorname{div} \tau
$$

where $P_{h}: L^{2}\left(\Omega, \mathbb{R}^{2}\right) \rightarrow V_{h}$ is the orthogonal projection, and $\operatorname{div}_{h}$ denotes the divergence operator defined locally on each triangle. A useful consequence of (3.5) is that $\operatorname{div}_{h} \Sigma_{h}=V_{h}$. Indeed, given any $v \in V_{h}$ we may find $\tau \in H^{1}(\Omega, \mathbb{S})$ such that $\operatorname{div} \tau=v$, and then $\Pi_{h} \tau \in \Sigma_{h}$ satisfies $\operatorname{div}_{h} \Pi_{h} \tau=P_{h} v=v$.

The piecewise-divergence-free elements of the space $\Sigma_{h}$ can also be explicitly characterized. On a triangle $T$ let

$$
Q_{T}=\left\{q \in \mathcal{P}_{4}(T, \mathbb{R})|\quad q|_{T} \in \mathcal{P}_{3}(e, \mathbb{R}) \quad \text { for each edge } e \text { of } T\right\} .
$$

This space has dimension 12 and any element $q \in Q_{T}$ can be uniquely determined by the following degrees of freedom (see Figure 2):

- the values of $q$ and the two first order partial derivatives of $q$ at each vertex ( 9 degrees of freedom)

- the values of the moment of degree 0 of the normal derivative of $q$ on each edge $e$ of $T$ (3 degrees of freedom)

The associated finite element space $Q_{h}$, defined with respect to the triangulation $\mathcal{T}_{h}$, is a subspace of $H^{1}(\Omega)$, but not a subspace of $H^{2}(\Omega)$, since the normal derivative is not continuous across the edges. However, the normal derivative is weakly continuous on each edge, and the space $Q_{h}$ has been proposed as a nonconforming approximation of $H^{2}(\Omega)$, see [11].

It is straightforward to check that the piecewise Airy stress function, $J_{h}$, maps $Q_{h}$ into the space of piecewise divergence-free elements of $\Sigma_{h}$. The relationship between $Q_{h}$ and $\Sigma_{h}$ is even more intimate. Define a projection operator $I_{h}: C^{\infty}(\Omega) \rightarrow Q_{h}$ by requiring that the vertex values of $I_{h} q$, the vertex values of $\operatorname{grad} I_{h} q$, and the edge moments of degree 0 of $\partial\left(I_{h} q\right) / \partial n$ all be equal to the corresponding values for $q$. It follows that on each edge $e$ of the triangulation

$$
\int_{e} \operatorname{grad}\left(q-I_{h} q\right) d s=\int_{e} \frac{\partial \operatorname{grad}}{\partial s}\left(q-I_{h} q\right) d s=0,
$$

whence also $\int_{T} J\left(q-I_{h} q\right) d x=0$ for all triangles $T$. From this the commutativity property $J_{h} I_{h} q=\Pi_{h} J q$ can be verified easily. We may then count dimensions to see that the second 

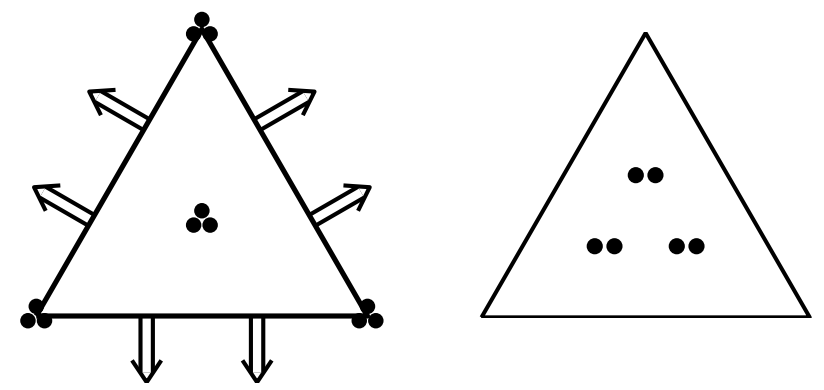

FigurE 3. Element diagrams for the stress and displacement of the lowest order conforming element.

row of the following diagram is exact (and hence the diagram is commuting with exact rows).

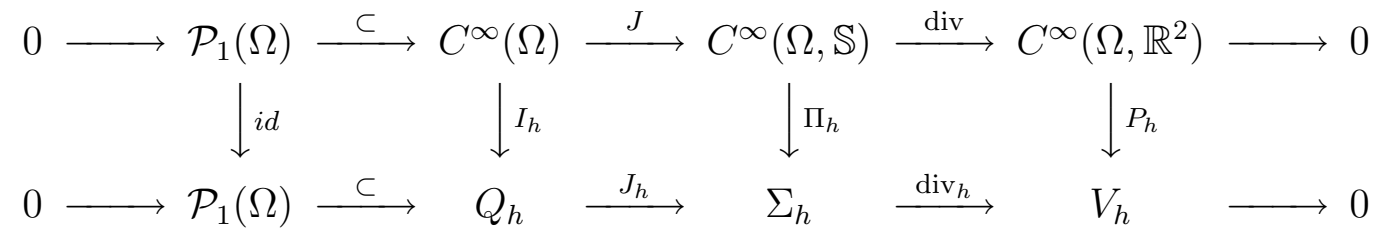

It is instructive to compare the nonconforming finite element space $\Sigma_{h}$ introduced above, with a low order conforming space we introduced in [5]. As recalled earlier, the conforming stress space consists of piecewise cubic matrix fields, with piecewise linear divergence and normal components continuous across each edge, and is paired with the same displacement space, namely the space of all piecewise linear vectorfields. Hence, locally on each triangle the space of stresses has dimension 24, as compared to 15 in the nonconforming case above. An element diagram for the conforming spaces is in given in Figure 3, where the three bullets at each vertex indicates that the three components of the stress field should be specified there. Comparing Figures 1 and 3, we see that the only the difference in degrees of freedom between the conforming and nonconforming case is that the vertex degrees of freedom have been removed in the latter case.

\section{THE NONCONFORMING MIXED METHOD AND ITS ERROR ANALYSIS}

The nonconforming mixed finite element method we propose to discretize (1.2) reads:

Find $\left(\sigma_{h}, u_{h}\right) \in \Sigma_{h} \times V_{h}$ such that

$$
\begin{gathered}
\int_{\Omega}\left(A \sigma_{h}: \tau+\operatorname{div}_{h} \tau \cdot u_{h}\right) d x=0, \quad \tau \in \Sigma_{h}, \\
\int_{\Omega} \operatorname{div}_{h} \sigma_{h} \cdot v d x=\int_{\Omega} f \cdot v d x, \quad v \in V_{h} .
\end{gathered}
$$

It is easy to see that this system has a unique solution. Indeed, if $f=0$, then we may take $\tau=\sigma_{h}$ and $v=u_{h}$ and subtract to conclude that $\sigma_{h}=0$, and then use the surjectivity of $\operatorname{div}_{h}: \Sigma_{h} \rightarrow V_{h}$ to conclude that $u_{h}=0$.

Key to the analysis of the new nonconforming finite element method are the properties of the interpolation operators $\Pi_{h}$ and $P_{h}$ mapping into $\Sigma_{h}$ and $V_{h}$, respectively. Note that the interpolation operator $\Pi_{h}$ is local with respect to triangulation $\mathcal{T}_{h}$, and locally it preserves 
linear matrix fields. Hence, using the matrix Piola transformation and a standard scaling argument, we obtain

$$
\left\|\Pi_{h} \tau-\tau\right\|_{0} \leq c h^{m}\|\tau\|_{m}, \quad 1 \leq m \leq 2
$$

See [5] for details. In particular we conclude that

$$
\left\|\Pi_{h} \tau\right\|_{0} \leq c\|\tau\|_{1}
$$

We also note that for the orthogonal projection operator $P_{h}$ onto discontinuous piecewise linear vector field we have the obvious error estimates

$$
\left\|v-P_{h} v\right\|_{0} \leq c h^{m}\|v\|_{m}, \quad 0 \leq m \leq 2
$$

Note that the stability conditions (A1) and (A2) are easily verified. The former holds with $c_{1}=1$ since $\operatorname{div}_{h} \Sigma_{h} \subset V_{h}$, and the latter follows from (3.5) and (4.4) by invoking the fact that for each $v \in V_{h} \subset L^{2}\left(\Omega, \mathbb{R}^{2}\right)$ there is $\tau \in H^{1}(\Omega, \mathbb{S})$ such that $\|\tau\|_{1} \leq c\|v\|_{0}$ and $\operatorname{div}_{h} \Pi_{h} \tau=\operatorname{div} \tau=v$.

We now state and prove precise error estimates for the nonconforming method (4.1)-(4.2). The proofs will not be based on (A1) and (A2), but instead will appeal directly to the properties of the spaces and of the interpolation operators which imply these conditions.

Theorem 4.1. Let $(\sigma, u)$ be the unique critical point of the Hellinger-Reissner functional over $H(\operatorname{div}, \Omega, \mathbb{S}) \times L^{2}\left(\Omega, \mathbb{R}^{2}\right)$ and let $\left(\sigma_{h}, u_{h}\right) \in \Sigma_{h} \times V_{h}$ be unique solution of $(4.1)-(4.2)$, where $\Sigma_{h}$ and $V_{h}$ are the spaces defined in Section 3. Then

$$
\begin{gathered}
\left\|\sigma-\sigma_{h}\right\|_{0} \leq \operatorname{ch}\|u\|_{2} \\
\left\|\operatorname{div} \sigma-\operatorname{div} \sigma_{h}\right\|_{0} \leq h^{m}\|\operatorname{div} \sigma\|_{m}, \quad 0 \leq m \leq 2, \\
\left\|u-u_{h}\right\|_{0} \leq \operatorname{ch}\|u\|_{2}
\end{gathered}
$$

Proof. For the exact solution, $u \in H^{1}(\Omega, \mathbb{S})$ and $\epsilon u=A \sigma$. Multiplying by $\tau \in \Sigma_{h}$, integrating over each element by parts, and summing over the elements we find that

$$
\int_{\Omega}\left(A \sigma: \tau+\operatorname{div}_{h} \tau \cdot u\right) d x=E_{h}(u, \tau), \quad \tau \in \Sigma_{h}
$$

where the consistency error $E_{h}(u, \tau)$ is defined as

$$
E_{h}(u, \tau)=\sum_{e \in \mathcal{E}_{h}} \int_{e}[t \cdot \tau n] u \cdot t d s,
$$

with the sum taken over the set of all edges, $\mathcal{E}_{h}$, of the triangulation $\mathcal{T}_{h}$. Here we use the notation $[t \cdot \tau n]$ to denote the jump of the quantity $t \cdot \tau n$ across the edge $e$, i.e., the difference of the traces taken from the two triangles sharing the edge. From the equilibrium equation $\operatorname{div} u=f$, we immediately have

$$
\int_{\Omega} \operatorname{div} \sigma \cdot v d x=\int_{\Omega} f \cdot v d x, \quad v \in V_{h} .
$$


Subtracting (4.6)-(4.7) from this the discrete system (4.1)-(4.2), and using the fact that $\operatorname{div}_{h} \Sigma_{h} \subset V_{h}$, we obtain

$$
\begin{gathered}
\int_{\Omega}\left[A\left(\sigma-\sigma_{h}\right): \tau+\operatorname{div}_{h} \tau \cdot\left(P_{h} u-u_{h}\right)\right] d x=E_{h}(u, \tau), \quad \tau \in \Sigma_{h}, \\
\int_{\Omega}\left(\operatorname{div} \sigma-\operatorname{div}_{h} \sigma_{h}\right) \cdot v d x=0, \quad v \in V_{h} .
\end{gathered}
$$

The second equation immediately implies that

$$
\operatorname{div}_{h} \sigma_{h}=P_{h} \operatorname{div} \sigma=\operatorname{div}_{h} \Pi_{h} \sigma
$$

where the last equality comes from (3.5). Hence, from (4.5), we immediately obtain the desired error bound for $\operatorname{div} \sigma$ :

$$
\left\|\operatorname{div} \sigma-\operatorname{div}_{h} \sigma_{h}\right\|_{0}=\left\|\left(I-P_{h}\right) \operatorname{div} \sigma\right\|_{0} \leq c h^{m}\|\operatorname{div} \sigma\|_{m}, \quad 0 \leq m \leq 2 .
$$

Taking $\tau=\Pi_{h} \sigma-\sigma_{h}$ in (4.8) and invoking (4.10) we obtain

$$
\int_{\Omega} A\left(\sigma-\sigma_{h}\right):\left(\Pi_{h} \sigma-\sigma_{h}\right) d x=E_{h}\left(u, \Pi_{h} \sigma-\sigma_{h}\right)
$$

from which it easily follows that

$$
\left\|\sigma-\sigma_{h}\right\|_{A}^{2} \leq\left\|\sigma-\Pi_{h} \sigma\right\|_{A}^{2}+2 E_{h}\left(u, \Pi_{h} \sigma-\sigma_{h}\right),
$$

where $\|\tau\|_{A}^{2}:=\int A \tau: \tau d x$. Since the norm $\|\cdot\|_{A}$ is equivalent to the $L^{2}$-norm it follows from (4.3) that

$$
\left\|\sigma-\Pi_{h} \sigma\right\|_{A} \leq c h^{m}\|\sigma\|_{m}, \quad 1 \leq m \leq 2 .
$$

In order to estimate $E_{h}(u, \tau)$, for $\tau \in \Sigma_{h}$, let $e \in \mathcal{E}_{h}$ be an edge and $\phi \in \mathcal{P}_{2}(e)$ be given by $\phi=[t \cdot \tau n]$. Since $\phi$ has mean value zero on $e$ it follows that by a standard scaling argument that

$$
\int_{e} \phi u \cdot t d s \leq\|\phi\|_{0, e} \inf _{\mu \in \mathbb{R}}\|u \cdot t-\mu\|_{0, e} \leq c|e|^{1 / 2}\|\phi\|_{0, e}\|u\|_{1, T^{-} \cup T^{+}},
$$

where $|e|$ is the length of $e$ and $T^{-}$and $T^{+}$are the two triangles meeting $e$. Furthermore, by shape regularity and scaling we get that $\|\phi\|_{0, e} \leq c|e|^{-1 / 2}\|\tau\|_{0, T^{-} \cup T^{+}}$. Hence, we obtain the bound

$$
\left|E_{h}(u, \tau)\right| \leq c\|\tau\|_{0}\|u\|_{1}, \quad u \in H^{1}\left(\Omega, \mathbb{R}^{2}\right), \tau \in \Sigma_{h} .
$$

In addition, $E_{h}(v, \tau)=0$ for any continuous piecewise linear vector field $v$. Therefore, we obtain the estimate

$$
E_{h}(u, \tau)=E_{h}\left(u-u_{h}^{I}, \tau\right) \leq c h\|\tau\|_{0}\|u\|_{2},
$$

where $u_{h}^{I}$ is the interpolant of $u$ into continuous piecewise linears. From (4.12) and (4.13) we can therefore conclude that

$$
\left\|\sigma-\sigma_{h}\right\| \leq \operatorname{ch}\left(\|\sigma\|_{1}+\|u\|_{2}\right) \leq \operatorname{ch}\|u\|_{2} .
$$

In order to establish the error estimate for the displacement, we recall that

$$
\left\|u-P_{h} u\right\|_{0} \leq c h^{2}\|u\|_{2} .
$$

Therefore, the desired estimate will follow from the bound

$$
\left\|P_{h} u-u_{h}\right\|_{0} \leq c h\|u\|_{2} .
$$



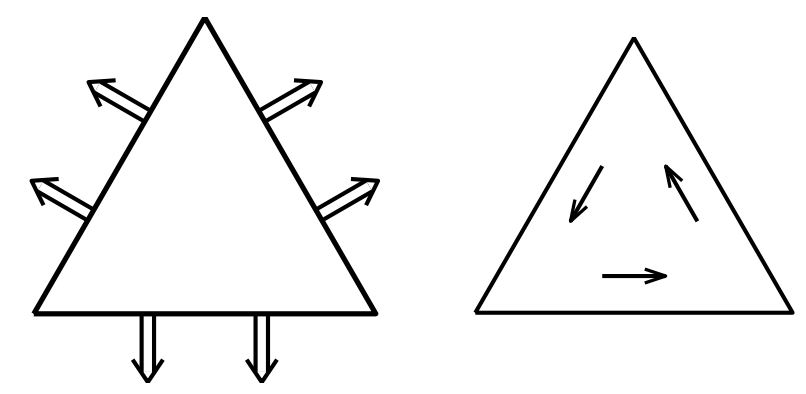

FiguRE 4. Element diagrams for the reduced nonconforming element.

Let $\tau \in H^{1}(\Omega, \mathbb{S})$ satisfy $\operatorname{div} \tau=P_{h} u-u_{h}$ with $\|\tau\|_{1} \leq c\left\|P_{h} u-u_{h}\right\|_{0}$. Then, in light of the commutativity property (3.5) and the bound (4.4), $\operatorname{div}_{h} \Pi_{h} \tau=P_{h} u-u_{h}$ and $\left\|\Pi_{h} \tau\right\|_{0} \leq$ $c\left\|P_{h} u-u_{h}\right\|_{0}$. Hence, using (4.1), (4.6), and the estimate (4.14),

$$
\begin{aligned}
\left\|P_{h} u-u_{h}\right\|_{0}^{2} & =\int_{\Omega} \operatorname{div}_{h} \Pi_{h} \tau \cdot\left(P_{h} u-u_{h}\right) d x \\
& =-\int_{\Omega} A\left(\sigma-\sigma_{h}\right): \Pi_{h} \tau d x+E_{h}\left(u, \Pi_{h} \tau\right) \\
& \leq c\left(\left\|\sigma-\sigma_{h}\right\|_{0}+h\|u\|_{2}\right)\left\|P_{h} u-u_{h}\right\|_{0} .
\end{aligned}
$$

Thus

$$
\left\|P_{h} u-u_{h}\right\|_{0} \leq c\left(\left\|\sigma-\sigma_{h}\right\|_{0}+h\|u\|_{2}\right) \leq c h\|u\|_{2}
$$

establishing (4.15).

\section{A SIMPLIFIED ELEMENT}

In the final section of the paper we briefly discuss a simplification of the nonconforming element $\left(\Sigma_{h}, V_{h}\right)$ studiesd above. The reduced velocity space, $\hat{V}_{h}$, will only contain discontinuous piecewise rigid motions, i.e., $\hat{V}_{T}=R M(T)$, where $R M(T)$ is the span of $\mathcal{P}_{0}\left(T, \mathbb{R}^{2}\right)$ and the linear vector field $\left(-x_{2}, x_{1}\right)$. The local stress space, $\hat{\Sigma}_{T}$, is given by

$$
\hat{\Sigma}_{T}=\left\{\tau \in \Sigma_{T} \mid \operatorname{div} \tau \in R M(T)\right\} .
$$

Now, the space of rigid motions is precisely the kernel of the symmetric gradient operator $\epsilon$. Therefore, a simple modification of the proof of Lemma 3.1 shows that $\operatorname{dim} \hat{\Sigma}_{T}=12$ and that a unisolvent set of degrees of freedom are given by the values of the moments of degree 0 and 1 of the two normal components of $\tau$ on each edge $e$ of $T$. An element diagram for the pair $\left(\hat{\Sigma}_{h}, \hat{V}_{h}\right)$ is given in Figure 4 .

The divergence-free elements of $\hat{\Sigma}_{h}$ will be the same as the divergence-free elements of $\Sigma_{h}$. Furthermore, the associated interpolation operators, $\hat{\Pi}_{h}$ and $\hat{P}_{h}$, still satisfies the commutativity property (3.5), and hence the obvious analog of the complete diagram (3.6) holds for the reduced spaces. Here the interpolation operator $\hat{\Pi}_{h}: H^{1}(\Omega, \mathbb{S}) \mapsto \hat{\Sigma}_{h}$ is defined by the degrees of freedom as above, while $\hat{P}_{h}: L^{2}\left(\Omega, \mathbb{R}^{2}\right) \mapsto V_{h}$ is the orthogonal projection. Since $\hat{\Pi}_{h}$ preserves linear matrix fields locally, the estimates (4.3) and (4.4) still holds for $\hat{\Pi}_{h}$. However, we should note that the space $\hat{\Sigma}_{T}$ is not invariant under Piola transforms. Therefore, a different scaling argument is required to prove these properties, for example by 
scaling to a similar element of unit diameter using translation, rotation, and dilation, and using a compactness argument.

Since the $\mathcal{P}_{0}\left(T, \mathbb{R}^{2}\right) \subsetneq \hat{V}_{T} \subsetneq \mathcal{P}_{1}\left(T, \mathbb{R}^{2}\right)$ the projection operator $\hat{P}_{h}$ will only allow a first order approximation in $L^{2}$, i.e. the estimate (4.5) is reduced to

$$
\left\|v-P_{h} v\right\|_{0} \leq c h^{m}\|v\|_{m}, \quad 0 \leq m \leq 1 .
$$

By following closely the derivation of the previous section these interpolation errors leads to the following error estimates for the reduced method:

$$
\begin{gathered}
\left\|\sigma-\sigma_{h}\right\|_{0} \leq \operatorname{ch}\|u\|_{2}, \\
\left\|\operatorname{div} \sigma-\operatorname{div} \sigma_{h}\right\|_{0} \leq c h^{m}\|\operatorname{div} \sigma\|_{m}, \quad 0 \leq m \leq 1, \\
\left\|u-u_{h}\right\|_{0} \leq \operatorname{ch}\|u\|_{2} .
\end{gathered}
$$

We observe that, compared to the estimates given in Theorem 4.1 for the non-reduced method, only the estimate for the error in the divergence have been weakend, while the size of the discrete system has been reduced by approximately $40 \%$.

\section{REFERENCES}

[1] Mohamed Amara and Jean-Marie Thomas, Equilibrium finite elements for the linear elastic problem, Numer. Math. 33 (1979), no. 4, 367-383.

[2] Douglas N. Arnold, Franco Brezzi, and Jim Douglas, Jr., PEERS: a new mixed finite element for plane elasticity, Japan J. Appl. Math. 1 (1984), no. 2, 347-367.

[3] Douglas N. Arnold, Jim Douglas, Jr., and Chaitan P. Gupta, A family of higher order mixed finite element methods for plane elasticity, Numer. Math. 45 (1984), no. 1, 1-22.

[4] Douglas N. Arnold and Richard S. Falk, A new mixed formulation for elasticity, Numer. Math. 53 (1988), no. 1-2, 13-30.

[5] Douglas N. Arnold and Ragnar Winther, Mixed finite elements for elasticity, to appear in Numer. Math.

[6] Franco Brezzi, On the existence, uniqueness and approximation of saddle-point problems arising from Lagrangian multipliers, Rev. Française Automat. Informat. Recherche Opérationnelle Sér. Rouge 8 (1974), no. R-2, 129-151.

[7] Franco Brezzi and Michel Fortin, Mixed and Hybrid Finite Element Methods, Springer-Verlag, New York, 1991.

[8] Michael Eastwood, A complex from linear elasticity, Rend. Circ. Mat. Palermo (2) Suppl. (2000), no. 63, 23-29.

[9] Baudoiun M. Fraejis de Veubeke, Displacement and equilibrium models in the finite element method, Stress analysis, (O.C Zienkiewics and G.S. Holister, eds.), Wiley, New York, 1965, pp. 145-197.

[10] Claes Johnson and Bertrand Mercier, Some equilibrium finite element methods for two-dimensional elasticity problems, Numer. Math. 30 (1978), no. 1, 103-116.

[11] Trygve K. Nilsen, Xue-Cheng Tai, and Ragnar Winther, A robust nonconforming $H^{2}$-element, Math. Comp. 70 (2001), no. 234, 489-505.

[12] Erwin Stein and Raimund Rolfes, Mechanical conditions for stability and optimal convergence of mixed finite elements for linear plane elasticity, Comput. Methods Appl. Mech. Engrg. 84 (1990), no. 1, 77-95.

[13] Rolf Stenberg, On the construction of optimal mixed finite element methods for the linear elasticity problem, Numer. Math. 48 (1986), no. 4, 447-462.

[14] _ A family of mixed finite elements for the elasticity problem, Numer. Math. 53 (1988), no. 5, $513-538$.

[15] _ . Two low-order mixed methods for the elasticity problem, The mathematics of finite elements and applications, VI (Uxbridge, 1987), Academic Press, London, 1988, pp. 271-280.

[16] Vernon B. Watwood Jr. and B. J. Hartz, An equilibrium stress field model for finite element solution of two-dimensional elastostatic problems, Internat. Jour. Solids and Structures 4 (1968), 857-873. 
Institute for Mathematics and its Applications, University of Minnesota, Minneapolis, MN 55455

E-mail address: arnold@ima.umn.edu

$U R L$ : http://www.ima.umn.edu/ arnold

Department of Informatics and Department of Mathematics, University of Oslo, P.O. Box 1080, Blindern, 0316 Oslo, Norway

E-mail address: rwinther@ifi.uio.no

URL: http://www.ifi.uio.no/ winther/index_eng.html 